\title{
Exometabolomic Profiling of Bacterial Strains as Cultivated Using Arabidopsis Root Extract as the Sole Carbon Source
}

\author{
Richard P. Jacoby, ${ }^{\dagger}$ Anna Martyn, and Stanislav Kopriva \\ Botanical Institute, Cluster of Excellence on Plant Sciences (CEPLAS), University of Cologne, D-50674 Cologne, Germany
}

Accepted 15 February 2018.

\begin{abstract}
The ability of microorganisms to use root-derived metabolites as growth substrates is a key trait for success in the rhizospheric niche. However, few studies describe which specific metabolites are consumed or to what degree microbial strains differ in their substrate consumption patterns. Here, we present a liquid chromatography-mass spectrometry (MS) exometabolomic study of three bacterial strains cultivated using either glucose or Arabidopsis thaliana root extract as the sole carbon source. Two of the strains were previously isolated from field-grown Arabidopsis roots, the other is Escherichia coli, included as a comparison. When cultivated on root extract, a set of $62 \mathrm{MS}$ features were commonly taken up by all three strains, with $m / z$ values matching components of central metabolism (including amino acids and purine or pyrimidine derivatives). Escherichia coli took up very few MS features outside this commonly consumed set, whereas the root-inhabiting strains took up a much larger number of MS features, many with $\mathrm{m} / \mathrm{z}$ values matching plantspecific metabolites. These measurements define the metabolic niche that each strain potentially occupies in the rhizosphere. Furthermore, we document many MS features released by these strains that could play roles in cross-feeding, antibiosis, or signaling. We present our methodological approach as a foundation for future studies of rhizosphere exometabolomics.
\end{abstract}

The rhizosphere (here widely defined to include plant roots and the immediately surrounding soil) contains a far higher concentration of microbes compared with bulk soil. This is primarily because the rhizosphere is rich in labile carbon, both within root cells and released into the soil via rhizodeposition. This stimulates the growth of soil microbes, which are usually carbon-limited (Dennis et al. 2010; Hartmann et al. 2009). However, the taxonomic breakdown of the rhizospheric microbiome is substantially different from the soil microbiome (Bulgarelli et al. 2013; Lundberg et al. 2012), indicating that certain strains are better adapted to colonize the rhizospheric niche. Studies

${ }^{\dagger}$ Corresponding author: Richard Jacoby; E-mail: rjacoby@uni-koeln.de

Funding: R. P. Jacoby is funded by the Horizon 2020 Marie Curie Sklodowska Action project 705808-PINBAC and research in the S. Kopriva lab is funded by the Deutsche Forschungsgemeinschaft grant EXC 1028.

*The $\boldsymbol{e}$-Xtra logo stands for "electronic extra" and indicates that four supplementary figures and four supplementary tables are published online.

This article is in the public domain and not copyrightable. It may be freely reprinted with customary crediting of the source. The American Phytopathological Society, 2018. have investigated which particular traits are important for microbial rhizosphere competence, highlighting mechanisms such as chemotaxis, antibiosis, and biofilm formation (Barret et al. 2011; Compant et al. 2010). But at a metabolic level, perhaps the most important factor determining rhizosphere competence is the ability to effectively use root-derived metabolites as a carbon source for growth (Benizri et al. 2002; Knee et al. 2001).

The taxonomic composition of the rhizosphere microbiome has been intensively studied over the last 15 years. Using bacterial $16 \mathrm{~S}$ pyrosequencing, studies almost invariably report a set of core bacterial taxa that are detected in the rhizosphere, including actinobacteria, flavobacteria, alpha-, beta-, and gammaproteobacteria, firmicutes, and flavobacteria (Bulgarelli et al. 2013; Müller et al. 2016). The consistent emergence of taxonomic diversity in the rhizosphere suggests that multiple strains can coexist in the same environment, facilitated via niche differentiation processes. One aspect of niche differentiation involves strains occupying different physical locations in the rhizosphere, for instance certain strains colonizing the root surface, followed by a subset of these strains subsequently proceeding to colonize the interior of the root (Beckers et al. 2017; Edwards et al. 2015; McCully 2001). But given the huge diversity of metabolites found in plant tissue, another important process driving rhizosphere microbial diversity is resource partitioning at the metabolic level, whereby individual bacterial strains are each specialized to consume discrete subsets of rootderived metabolites (Goldfarb et al. 2011).

There is ongoing investigation as to whether different plant genotypes can reproducibly recruit different microbial strains to their rhizospheric microbiomes. Although soil and climate seem to exert dominant influences upon the taxonomic composition of the rhizosphere microbiome, there is evidence that plant genotype also exerts a significant effect (Bulgarelli et al. 2015; Peiffer et al. 2013). Mechanistically, it is particularly interesting to define the specific plant-derived molecules that are involved in the recruitment of microbial strains. There is extensive literature implicating flavonoids as a key player in plant-microbe interactions, particularly for recruitment of nitrogenfixing rhizobium strains to legume root nodules (Hassan and Mathesius 2012). Furthermore, soil incubated with purified flavonoids exhibited an altered microbial community structure (Szoboszlay et al. 2016), indicating that flavonoids can affect the growth behavior of a wide range of soil microbes. Therefore, it can be hypothesized that flavonoids are a key player in the chemical dialogue used by plants to recruit specific microbial strains from the soil and into their rhizosphere microbiome.

In the microbiology literature, there is a long tradition of studying which carbon sources are utilized by bacterial strains as growth substrates (Harder and Dijkhuizen 1982; Monod 1966). 
Methodologically, classical studies involved providing a single substrate or a simple mixture of substrates and using biochemical methods to measure the depletion of individual metabolites from the medium. The limitation of this approach is the low throughput of measurement, meaning that relatively few substrates can be provided to the organism simultaneously. Another approach involves phenotype microarrays, where microbial strains are incubated in 96-well plates in which each well containing a single growth substrate and growth is monitored over time (Bochner et al. 2001). The disadvantage of this method is that the plates are loaded with a predefined set of growth substrates, which cannot fully represent the diverse array of metabolites present in the rhizosphere. Recently, studies investigating microbial substrate preferences are increasingly beginning to incorporate highthroughput exometabolomic approaches, in which nuclear magnetic resonance (NMR) or mass spectrometry (MS) are used to measure which metabolites are depleted from the growth medium during microbial growth (Silva and Northen 2015). The advantage of this approach is that the consumption of several hundred metabolites can be measured in complex media, meaning that the media can be formulated to reflect the potential growth substrates available to the organism in the rhizosphere.

Exometabolomic approaches have been applied to investigate metabolite uptake and release across a wide range of organisms, with an increasing tendency to design experimental scenarios that represent the metabolic milieu of the organism's natural habitat. Baran et al. (2015) presents a study of metabolic crossfeeding among soil crust isolates, reconstructing a simplified community comprised of one autotrophic primary producer and five heterotrophic consumers. Using liquid chromatography (LC)-MS exometabolomics, the study found strong evidence for metabolic resource partitioning among the heterotrophic strains, with each strain consuming a different subset of available metabolites. In the phyllosphere, Ryffel et al. (2016) inoculated Arabidopsis leaves with three different bacterial strains and conducted NMR metabolomics on a solution derived from rinsing the leaves as well as MS imaging of the leaves themselves. This revealed that nucleotide metabolites colocalize with bacterial colonization sites and, also, that the strains appear to modulate the plant-derived arginine pool. Regarding rhizospheric strains, Fei et al. (2016) presents an exometabolomic study of nitrogen-fixing legume symbiont Sinorhizobium meliloti strains grown on laboratory medium, showing that deletion of the pSymB plasmid results in slower consumption of several amino acids. However, to our knowledge there are no studies using exometabolomic approaches to investigate bacterial substrate utilization from a growth medium that includes plant root extracts.

In this study, we conducted an exometabolomic analysis of individual bacterial strains, cultivated using either glucose or Arabidopsis thaliana root extracts as the sole carbon source in the growth medium. We aimed to determine which root-derived metabolites are consumed by each strain and which metabolites the strains excrete into the medium. Also, we investigate the metabolomic footprint of strains grown using the flavonoiddeficient $t t 4$ Arabidopsis mutant. The aim of our work was not to create a complete list of metabolites but, rather, to test the suitability of this methodological approach as a foundation for future comprehensive studies of rhizosphere exometabolomics.

\section{RESULTS}

\section{Experimental workflow for exometabolomic analysis using root extract as the sole carbon source.}

Here, we present a novel methodology for studying which root-derived metabolites are consumed by individual bacterial strains; a schematic of the experimental workflow is presented in Supplementary Figure S1. First, large quantities of Arabidopsis root tissue are produced using the method of Hétu et al. (2005). In our hands, this procedure yielded approximately $1.5 \mathrm{~g}$ of root material per 250-ml conical flask. Next, metabolites are extracted from ground root material and the total organic carbon concentration of this metabolite extract is determined. Our extracts contained roughly $3 \mathrm{mg}$ of carbon per $1 \mathrm{~g}$ of fresh root tissue extracted. Bacterial growth media were then formulated, containing either glucose or root extract as the sole carbon source. The carbon concentration of all growth media was set to $0.72 \mathrm{mg}$ of $\mathrm{C}$ per milliliter of medium. At this concentration, carbon is the stoichiometrically limiting nutrient, as determined experimentally (Egli 2015) (Supplementary Fig. S2). Next, washed bacterial cells are inoculated into these growth media on a 48-well plate and bacterial growth is monitored via measurement of optical density at $600 \mathrm{~nm}\left(\mathrm{OD}_{600}\right)$ in a plate reader. Analysis of growth curves found that Arabidopsis root extract could support growth of all tested strains (Supplementary Fig. S3). After $24 \mathrm{~h}$, all cultures had reached stationary phase and spent media were harvested by separating bacterial cells via centrifugation, followed by collection and filtration of supernatants. The metabolomic composition of these spent-media samples were then analyzed using LC-MS and the resulting data was analyzed using XCMS online (Tautenhahn et al. 2012).

Global analysis of exometabolomic profiles using LC-MS.

First, we aimed to characterize how the exometabolome profile can be shaped by different bacterial strains and carbon sources by conducting a global analysis of metabolite peaks in the spent-media samples, measured using LC-MS. We analyzed three bacterial strains, Pseudomonas sp. Root 9 and Rhizobium sp. Root491 (both isolated from Arabidopsis roots by Bai et al. [2015]) and $E$. coli isolated from human gut and included to contrast against the rhizosphere strains. As a negative control (without bacteria), we also included samples in which the bacterial inoculation was replaced by sterile $\mathrm{MgCl}_{2}$ and incubated side-by-side with the bacterial cultures. Alignment of MS features between samples yielded 2,786 discrete MS features, which was reduced to 2,456 when isotopic peaks were removed. To highlight features that were significantly different between samples, we filtered the data to select 1,982 features with $P<0.01$ following $t$ test between any two groups (Supplementary Table S1) and arranged these features by hierarchical clustering (Fig. 1). Correction for multiple comparisons was conducted using the method of Storey (2002) and showed that $1,958 \mathrm{MS}$ features were significant at $q<0.01$. By looking at the resultant heatmap, it can be clearly seen that there is a large number of MS features present in the plant root samples but absent in the glucose samples, resulting in the dendrogram splitting the eight samples into two groups according to carbon source. Looking at the different rows in the heatmap, there is clear evidence for metabolite uptake from the growth media (when a feature showed higher abundance in $\mathrm{MgCl}_{2}$ and lower abundance in one or more bacterial inoculation) and also metabolite release into the growth media (when a feature has lower abundance in $\mathrm{MgCl}_{2}$ and higher abundance in one or more bacterial inoculation). Also evident is a cluster of MS features that are more abundant in the glucose media compared with the Col-0 media but not taken up by any strain. We believe that these are salt adducts derived from the M9 salts, which are masked by ion suppression in the Col-0 samples, because they elute at the same retention time (RT) as many charged metabolites, including major sugars. Noticeably, there was a very large cluster of MS features that were more abundant in samples in which root extract was the sole carbon source but which were not taken up by any of the three strains tested here. It would be interesting to determine whether these MS features can serve as growth substrates for other microbial strains not included in this study. 


\section{MS features taken up by bacterial strains}

when Arabidopsis root extract is the sole carbon source.

Our next goal was to determine how many MS features are consumed by the three strains when cultivated on Arabidopsis root extract as the sole carbon source and also the degree of overlap in these substrate uptake patterns between bacterial strains. Therefore, we filtered for MS features that were significantly depleted in the Col-0 medium inoculated with bacteria compared with Col- 0 medium incubated with $\mathrm{MgCl}_{2}$ (details of filtering provided below). This resulted in $422 \mathrm{MS}$ features that were implicated as root metabolites consumed by bacterial uptake, presented as a heat map and Venn diagram in Figure 2. From this analysis, we observed a core set of $62 \mathrm{MS}$ features taken up by all three strains. It was clear that Rhizobium sp. Root 491 consumes a much larger set of metabolites than the other two strains, with 372 MS features being consumed by this strain, compared with 187 in Pseudomonas sp. Root 9 and only 70 in E. coli. There was also a set of $76 \mathrm{MS}$ features commonly taken up by the two root-inhabiting strains but not taken up by $E$. coli.

\section{MS features released by bacterial strains} on either glucose or root extract as the sole carbon source.

We were also interested in characterizing the MS features that were excreted into the media by the different bacterial strains when either glucose or Col-0 root extract were provided as the sole carbon source. To select these features, we filtered for MS features that were substantially more abundant in the inoculated media compared with the media incubated with sterile $\mathrm{MgCl}_{2}$ control (details of filtering are provided below). The $448 \mathrm{MS}$ features that met filtering criteria are presented as a heatmap and several Venn diagrams in Figure 3. From these analyses, it is evident that there is relatively little overlap in the MS features released by an individual strain when grown on the two carbon sources. For instance, Rhizobium sp. Root491 releases 135 MS features uniquely when grown on Col-0 root extract and 65 only when grown on glucose, but the overlap between these two conditions contained just 19 MS features. For E. coli, only $48 \mathrm{MS}$ features were released under both conditions, whereas 147 were uniquely released under glucose nutrition.

\section{Assignment of candidate metabolite identity to MS features.}

Next, we wished to ascertain which particular metabolites correspond to the MS features that were taken up or released by the analyzed bacterial strains. Therefore, we used the Metabosearch program (Zhou et al. 2012) to assign candidate identities for the MS features that were taken up or released by the individual strains (Supplementary Table S2 for uptake, Supplementary Table S3 for release). For the 422 MS features that were taken up from Col-0 medium, Metabosearch gave candidate identities for 300, while for the 448 MS features that were released into either medium, Metabosearch gave candidate identities for 323. As a quality check for this approach, we began by assessing whether we could reliably detect the consumption of glucose in the cultures in which it was present as the sole carbon source. Therefore, we prepared a standard solution of $10 \mu \mathrm{M}$ glucose and measured this on our LC-MS system, to determine the best-responding reporter ion for this molecule $\left(\mathrm{Na}^{+}\right.$adduct of glucose, $\mathrm{m} / z$ 203.053, RT $\left.1.6 \mathrm{~min}\right)$. Then, we checked the abundance pattern of this particular MS feature in our spent-media samples, with the result shown in Figure 4. The huge depletion of glucose during bacterial growth can be observed by the low abundance of this MS feature in the three bacterial inoculations of the media in which glucose is the sole carbon source, compared against its very high abundance in the $\mathrm{MgCl}_{2}$ incubation. Although Metabosearch correctly identi- fied the molecular formula of this metabolite $\left(\mathrm{C}_{6} \mathrm{H}_{12} \mathrm{O}_{6}\right)$, our data filtering strategy led to this MS feature being assigned to fructose, an isomer of glucose. Furthermore, we also observed several other MS features exhibiting a very similar abundance pattern, eluting at the same RT, which almost certainly derive from different adducts and fragments of glucose. This highlights two caveats of our approach. i) Although the molecular formula assignment of a given MS feature is likely to be accurate due to the high mass accuracy of the MS system and the stringent search parameters used, the molecular structure of these assignations is uncertain because many isomers can derive from the same molecular formula. ii) The number of MS features detected may be higher than the number of discrete molecules in the sample, because one single molecule can be detected in multiple adducted or fragmented states.

\section{Metabolites of particular interest.}

Upon inspecting the derived data, we observed a number of candidate metabolites that warranted particular interest. First, we inspected the candidate identity of the $62 \mathrm{MS}$ features commonly taken up by all three strains, which revealed that

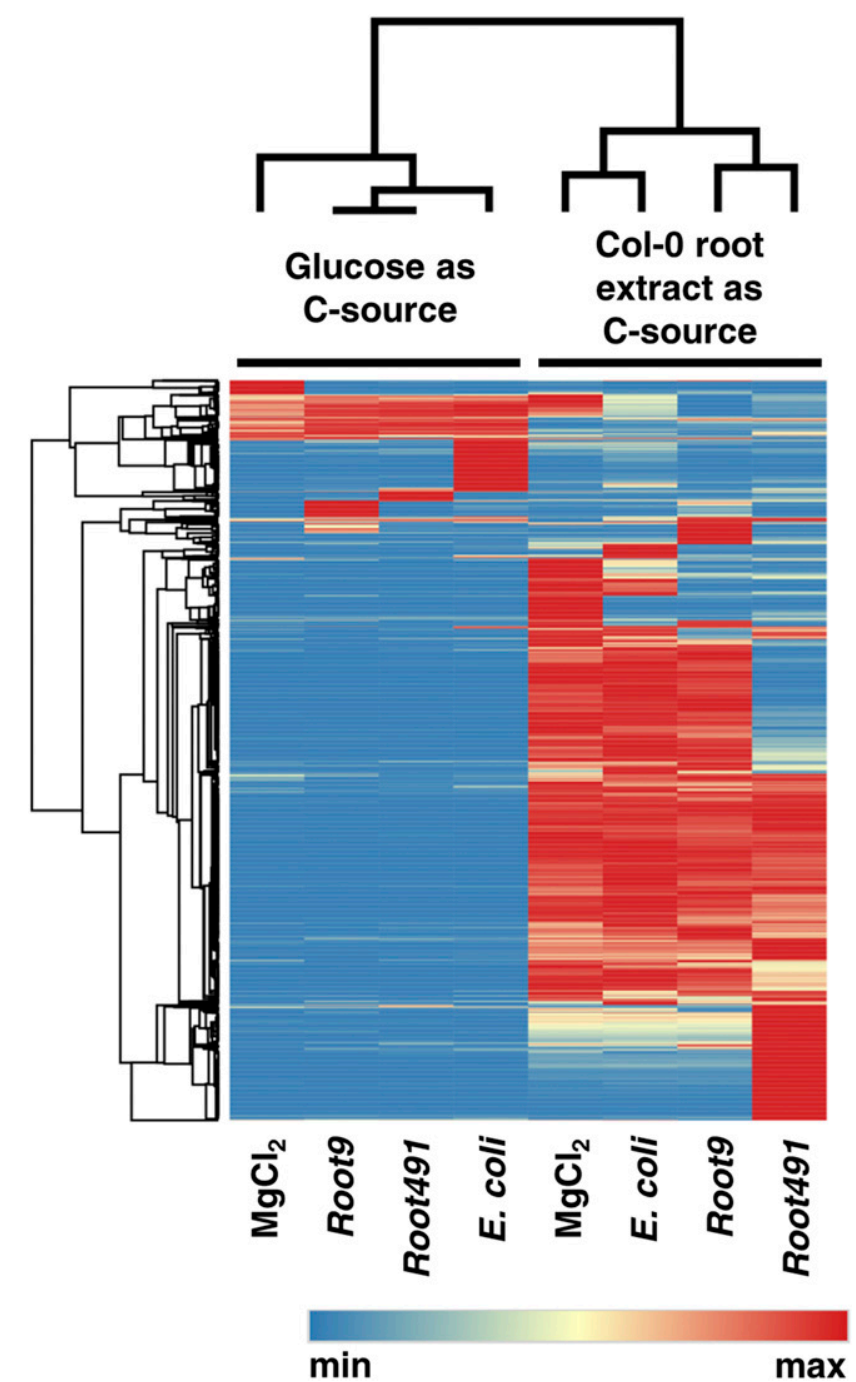

Fig. 1. Overview of exometabolomic differences observed between three bacterial strains cultivated on two carbon sources. The heatmap depicts the mean intensity values for the 1,982 mass spectral (MS) features that had a $P<0.01$ following $t$ test between any two sample groups. Each row corresponds to a MS feature and each column corresponds to a strain $\times$ medium combination. Rows and columns were hierarchically clustered using Pearson's correlation coefficient. Labels above the heatmap indicate carbon source, labels below the heatmap indicate bacterial inoculation, with $\mathrm{MgCl}_{2}$ as the sterile control. 
many of these features had $\mathrm{m} / \mathrm{z}$, values matching fundamental components of central metabolism, such as amino acids (including valine, tryptophan, threonine, and glutamine), and also purine or pyrimidine derivatives (including adenosine, cytidine, guanine, and uridine). In Figure 5, we show the abundance patterns for three of these metabolites that were taken up by all strains, annotated as tryptophan, uridine, and malate. Malate (Fig. 5C) is an interesting case, as it is consumed from the medium when Col- 0 root extract is the carbon source but released into the medium when glucose is the carbon source. Regarding metabolites that were released into the medium, we observed that $E$. coli excretes a large amount of orotate into the medium when grown under glucose as $\mathrm{C}$ source (Fig. 6A), which matches established literature (Enjalbert et al. 2013; Womack and O'Donovan 1978). For Pseudomonas sp. Root9, we detected the release of several nucleotides into the medium

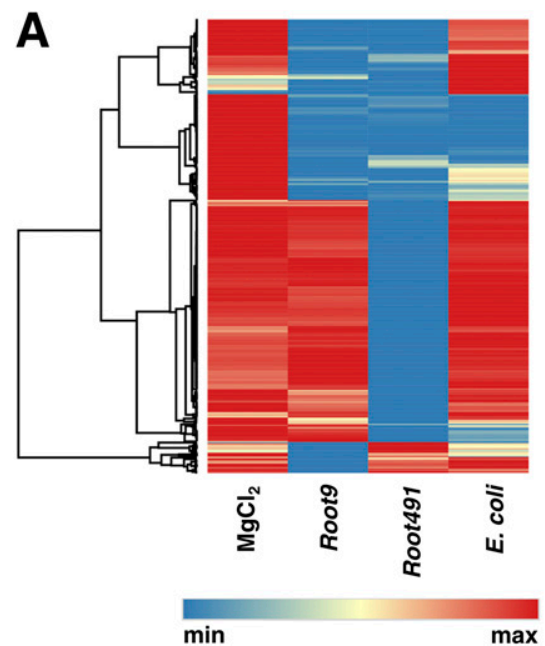

B

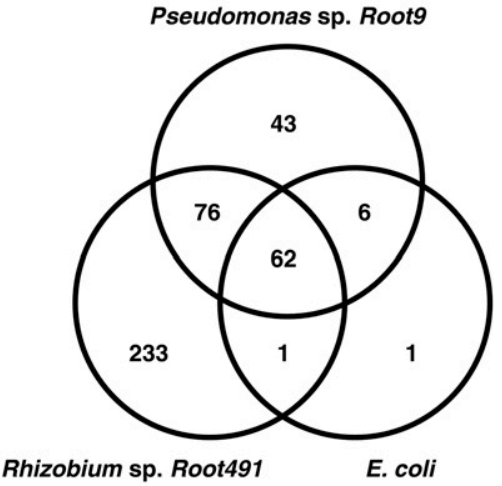

Fig. 2. Mass spectral (MS) features taken up by bacterial strains cultivated on medium containing Arabidopsis Col-0 root extract as the sole carbon source. A, A heatmap depicting the mean intensity values for the $422 \mathrm{MS}$ features that were taken up from Col- 0 medium by any bacterial strain. Each row corresponds to a MS feature and each column corresponds to a bacterial strain. Rows were hierarchically clustered using Pearson's correlation coefficient. Labels below the heatmap indicate bacterial inoculation, with $\mathrm{MgCl}_{2}$ as the sterile control. B, A Venn diagram showing the number of MS features that were taken up commonly and uniquely by the three strains.

A

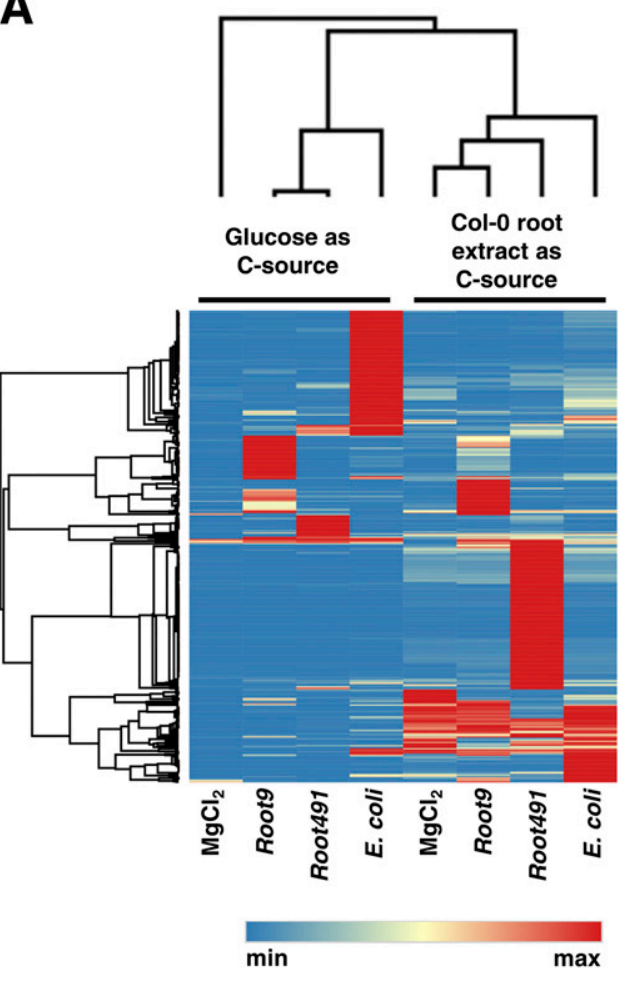

B

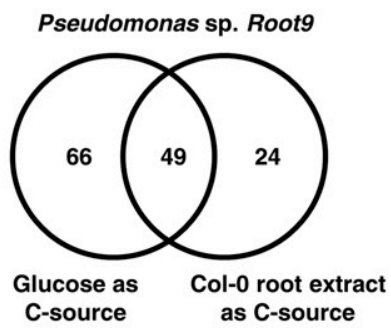

Rhizobium sp. Root491

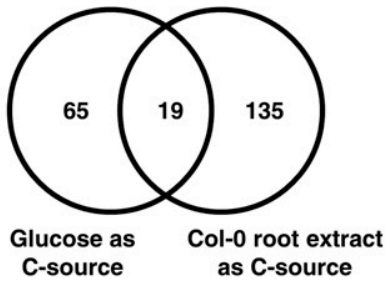

E. coli

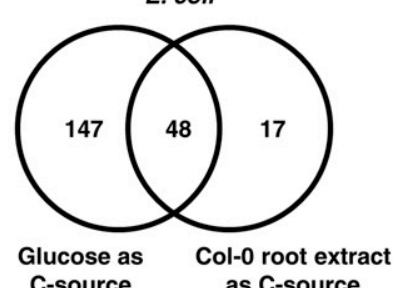

Fig. 3. Mass spectral (MS) features released by the bacterial strains. Data were filtered to select 448 MS features that were released into either glucose or Col0 medium by at least one strain. A, A heatmap depicting the mean intensity values for the $448 \mathrm{MS}$ features, in which each row corresponds to a MS feature and each column corresponds to a strain $\times$ medium combination. Rows and columns were hierarchically clustered using Pearson's correlation coefficient. $\mathrm{MgCl}_{2}$ was the sterile control. B, Venn diagrams showing the degree of overlap between MS features released by each strain across the two carbon sources. 
under both glucose and Col-0 nutrition, including $\mathrm{m} / \mathrm{z}$ values matching AMP, CMP, GMP, and UMP (Fig. 6B); these nucleotides could potentially play roles in signaling (Grundling and Lee 2016). Although we did not detect release of the active auxin indole-3-acetic acid by any of these strains, we did detect that Rhizobium sp. Root 491 releases an $\mathrm{m} / \mathrm{z}$ value matching an auxin conjugate, indole-3-acetyl-myo-inositol, under Col-0 nutrition but not glucose (Fig. 6C). It would be interesting to determine whether this bacterial-derived auxin conjugate can be taken up by the plant. For Rhizobium sp. Root491 grown under Col-0 root extract, we observed a particularly interesting phenomenon, whereby this strain took up MS features matching scopolin and sinapoyl glucose and released MS features matching scopoletin and sinapate (Fig. 7). We postulate that this strain can cleave and metabolize the labile sugar from these molecules but is unable to metabolize the more recalcitrant core structures.

\section{Application of our exometabolomic profiling approach to the flavonoid-deficient $\boldsymbol{t t} \boldsymbol{t}$ mutant.}

Finally, we investigated whether our methodological approach could be used to profile an Arabidopsis mutant. There is extensive literature implicating flavonoids as a key player in plant-microbe interactions (Hassan and Mathesius 2012; Szoboszlay et al. 2016), so we prepared root extract from the flavonoid-deficient $t t 4$ mutant and used this as a carbon source for bacterial growth. The absence of flavonoid-derived pigments in the $t t 4$ root extracts was confirmed by photometric measurements across the UV range (Supplementary Fig. S4). When analyzing growth phenotypes using Growthcurver, we observed no significant differences between Col-0 and $t t 4$ root extracts, suggesting that flavonoids are not major metabolic substrates for the growth of any bacterial strains assayed here. Next, we compared exometabolomic profiles between Col0 medium versus the $t t 4$ medium, filtering for MS features that were significantly different between the two samples (Fig. 8A). This comparison yielded $62 \mathrm{MS}$ features, with the predominant grouping being $41 \mathrm{MS}$ features that were highly abundant in all four Col-0 samples but low abundance in all four $t t 4$ samples. Upon inspecting the candidate identities of these features derived from Metabosearch, we found that 16 of these features were matched to $\mathrm{H}^{+}$adducts of flavonoid glycosides and, also, eight features matched to $\mathrm{Na}^{+}$adducts of flavonoid glycosides.

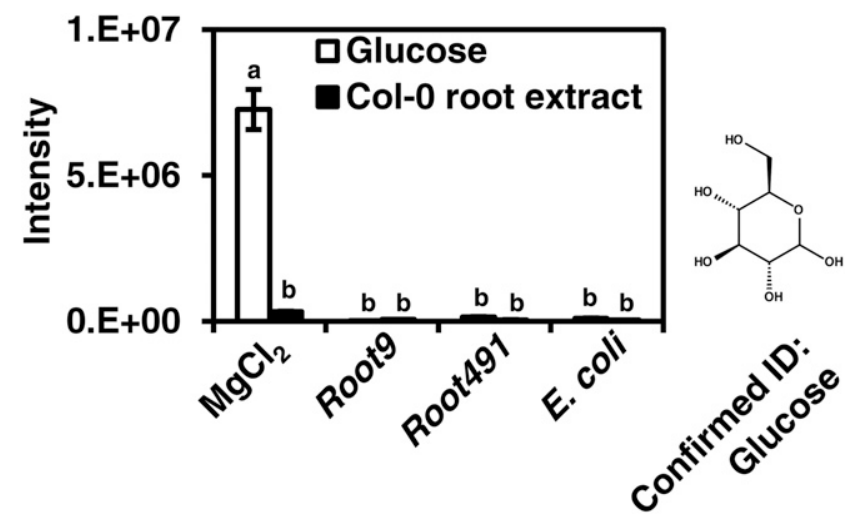

Fig. 4. Uptake of glucose by bacterial strains. White-filled columns represent mean intensity of the mass spectral (MS) feature when glucose was the sole carbon source, black-filled columns represent mean intensity of the MS feature when Col- 0 root extract was the sole carbon source. $X$ axis labels indicate bacterial inoculation, with $\mathrm{MgCl}_{2}$ as a sterile control. Error bars represent standard deviation $(n=3)$. Different letters indicate significant difference at $P<0.01$ (Tukey's highly significant difference test). Metabolite identity was confirmed by comparison with a reference glucose standard.
Looking at the abundance of these features in the samples where Col-0 root extracts had been inoculated with bacteria, we observed that none of the bacteria took up these candidate flavonoid glycosides (Fig. 8B and C). From this evidence, we conclude that flavonoid glycosides are not major metabolic substrates for these particular bacterial strains and, also, that exometabolomic profiles of these strains are not strongly influenced by flavonoid-linked signaling.

\section{DISCUSSION}

In the rhizosphere, there is a bidirectional exchange of nutrients between plants and microbes, but our mechanistic understanding
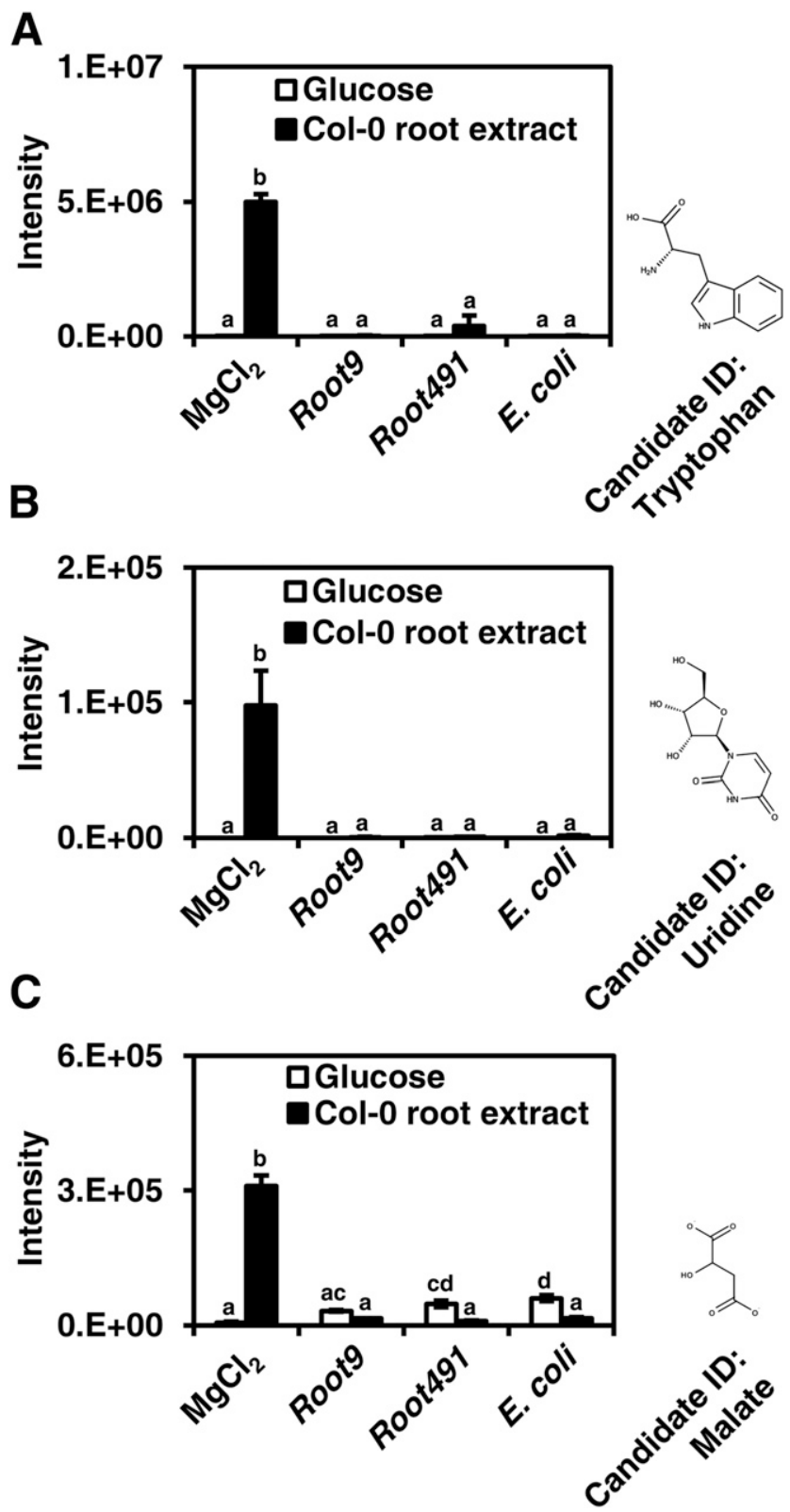

Fig. 5. Selected mass spectral features taken up by all three strains. Candidate metabolite identities (IDs) were matched to A, tryptophan, $\mathbf{B}$, uridine, and $\mathbf{C}$, malate. White-filled columns represent mean feature intensity when glucose was the sole carbon source, black-filled columns represent mean feature intensity when Col-0 root extract was the sole carbon source. $X$ axis labels indicate bacterial inoculation, with $\mathrm{MgCl}_{2}$ as a sterile control. Error bars represent standard deviation $(n=3)$. Different letters indicate significant difference at $P<0.01$ (Tukey's highly significant difference test). 
of this phenomenon is incomplete (Sasse et al. 2018). To gain new insights into this process, it will be important to develop new methodological approaches that can directly measure the metabolic activity of rhizospheric microbial strains. Exometabolomics is increasingly being used to profile metabolite uptake and release by various microbes (Pinu and Villas-Boas 2017), and here, we present the first report of MS exometabolomics applied to rhizospheric microbes using plant root extract as the sole carbon source. The presented approach is highly versatile, with the potential to analyze root extracts from any plant species or genotype and, also, to measure the metabolic footprint of any microbial population, including bacteria,
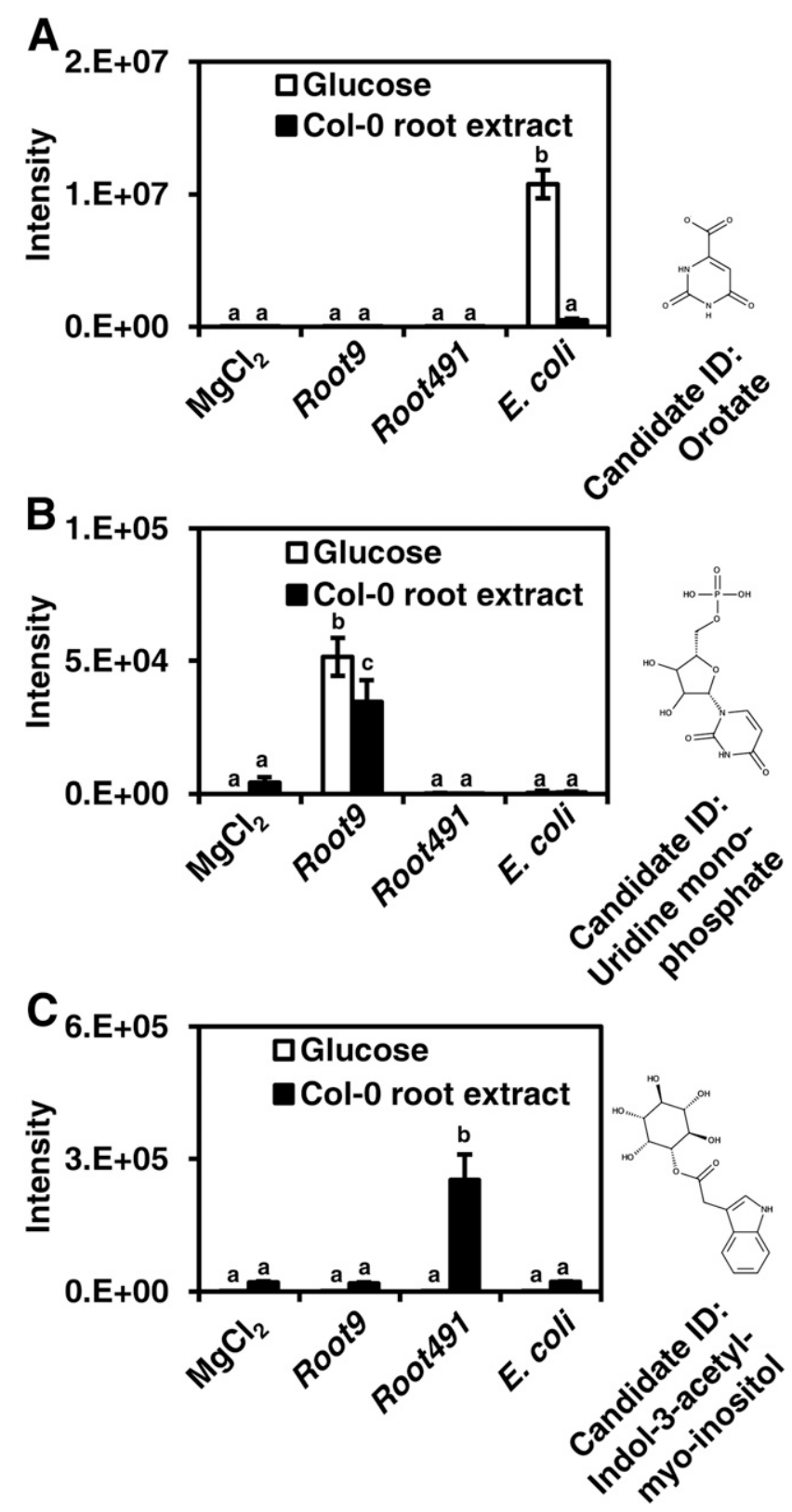

Fig. 6. Selected mass spectral features released by individual strains. Candidate metabolite identities (IDs) matched to $\mathbf{A}$, orotate, $\mathbf{B}$, uridine monophosphate, and $\mathbf{C}$, indol-3-acetyl-myo-inositol. White-filled columns represent mean feature intensity when glucose was the sole carbon source, black-filled columns represent mean feature intensity when Col-0 root extract was the sole carbon source. $X$ axis labels indicate bacterial inoculation, with $\mathrm{MgCl}_{2}$ as a sterile control. Error bars represent standard deviation $(n=3)$. Different letters indicate significant difference at $P<0.01$ (Tukey's highly significant difference test). fungi, or even synthetic microbial communities. Furthermore, future studies could combine this general approach with a wide range of other analytical possibilities, including alternative chromatography methods, other MS systems, or NMR detection of metabolites. We present this study as a first investigation of rhizosphere exometabolomics, to serve as a foundation for future work.

We analyzed three bacterial strains, two root-inhabiting (Pseudomonas sp. Root 9 and Rhizobium sp. Root491) recently isolated from field-grown Arabidopsis roots by Bai et al. (2015) and E. coli, isolated from human gut and included as a comparison. Comparing the substrate uptake profiles between the three strains, we observed a small set of MS features commonly taken up by all three strains but found that the two rootinhabiting strains took up a much larger set of MS features that were not taken up by E. coli. Regarding metabolite release, we found that each individual strain profile of released metabolites was dramatically influenced by the available carbon source (glucose versus Arabidopsis root extract), for instance, with Rhizobium sp. Root491, a large number of MS features was released only when root extract was provided as the carbon source. Furthermore, we applied our methodological approach to the flavonoid-deficient $t t 4$ mutant, finding that $t t 4$ root extracts were deficient in several MS features likely to be flavonoid glycosides. However, we observed no discernible uptake of these MS features when the strains were grown on Col-0 root extract, indicating that none of the three studied strains take up flavonoid glycosides as growth substrates.

A major priority of this study was to define which specific root metabolites are consumed as growth substrates by various bacterial strains, thus defining the metabolic niche that these strains can potentially occupy in the rhizsophere. We found a core set of 62 MS features that all three strains deplete from the growth medium when Col-0 root extract was the sole carbon source. Assigning candidate identities to these MS features showed that many were matched to molecules of central metabolism, such as amino acids and purine or pyrimidine derivatives. Extrapolating to the rhizosphere, we can infer that there would be a strong competition for these shared metabolic substrates and that a strain such as E. coli would find it difficult to compete in the rhizospheric niche, because it can only take up this limited set of substrates. We were particularly interested in identifying root-derived metabolites that were specifically consumed by individual strains, which could contribute to rhizospheric niche differentiation via metabolic resource partitioning. We observed that Rhizobium sp. Root491 took up 233 MS features that were not taken up by the other two strains, and, inspecting the candidate identities (IDs) for these MS features, we observed several $\mathrm{m} / \mathrm{z}$ values matching plantspecific metabolites such as scopolin, coniferin, sinapoyl glucose, and xylobiose. Although the Rhizobium taxon is bestknown for the nitrogen-fixing symbionts associated with legumes, there is increasing evidence that strains from this taxon are highly successful at colonizing a wide range of plant species (Chi et al. 2005; García-Fraile et al. 2012). Therefore, perhaps the wide range of plant-specific metabolites taken up by Rhizobium sp. Root 491 observed in this study reflects its metabolic specialization to consume phytochemicals as growth substrates. For Pseudomonas sp. Root9, we observed that this strain took up 43 MS features that were not taken up by the other two strains, including $\mathrm{m} / \mathrm{z}$ values matching the purine breakdown products allantoic acid and uric acid. There is significant literature describing bacterial strains that can catabolize allantoic acid and uric acid (Vogels and Vander Drift 1976), and it is known that plants accumulate significant amounts of these molecules, increasing under stress (Werner and Witte 2011). So, although most of the literature on bacterial consumption of 
allantoic acid and uric acid focuses on the cycling of nitrogenous waste from animals, our data indicate that these molecules could also be involved in nutrient exchange processes in the rhizosphere.
There is growing awareness that bacterial metabolite excretion plays a key role in shaping microbial community structure, via processes such as cross-feeding, signaling, and antibiosis (Foster et al. 2017; Widder et al. 2016). In this study, we
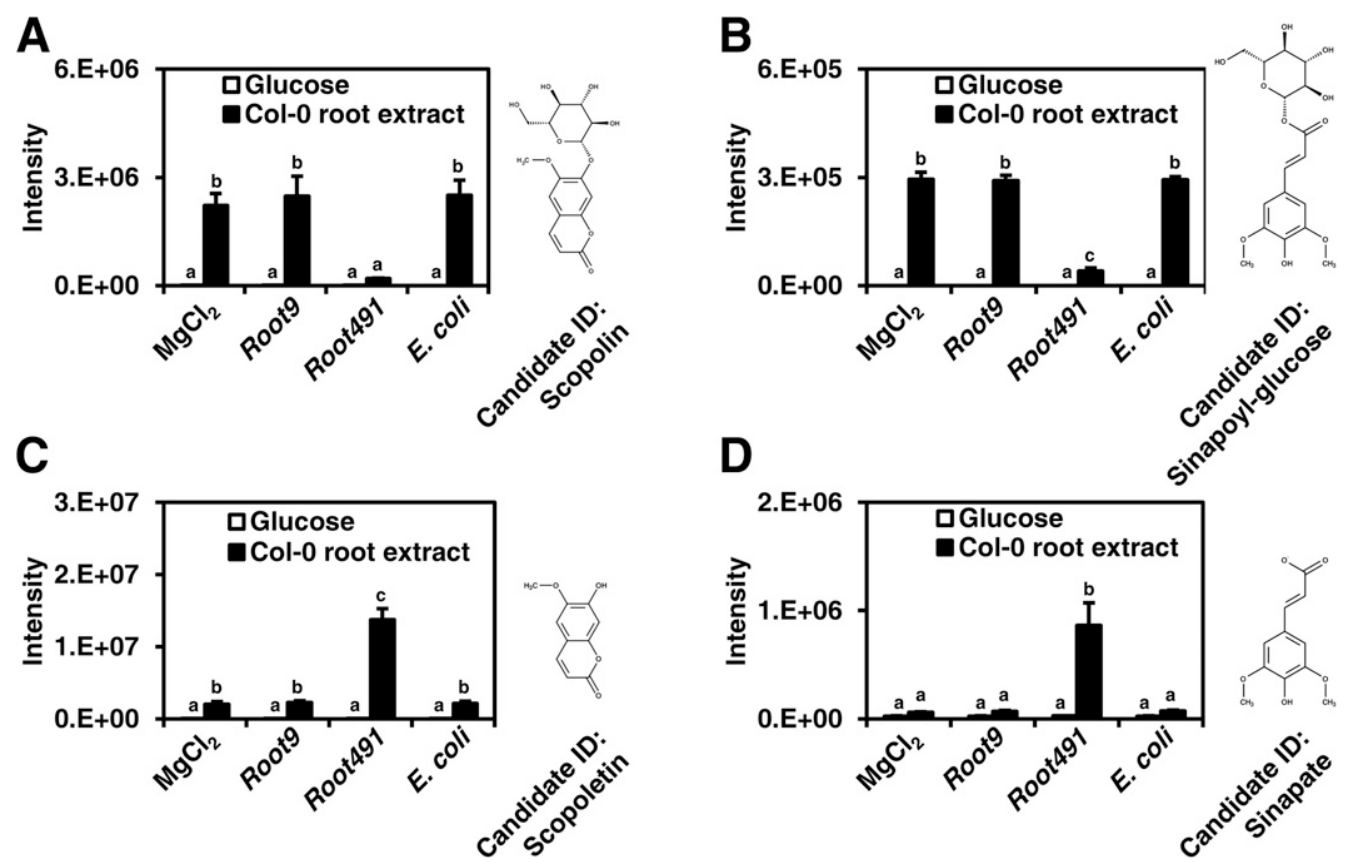

Fig. 7. Mass spectral features postulated to be reciprocally taken up and released by Rhizobium sp. Root491. Candidate metabolite identities (IDs) matched to A, scopolin, B, sinapoyl-glucose, C, scopoletin and D, sinapate. White-filled columns represent mean feature intensity when glucose was the sole carbon source, black-filled columns represent mean feature intensity when Col-0 root extract was the sole carbon source. $X$ axis labels indicate bacterial inoculation, with $\mathrm{MgCl}_{2}$ as a sterile control. Error bars represent standard deviation $(n=3)$. Different letters indicate significant difference at $P<0.01$ (Tukey's highly significant difference test).
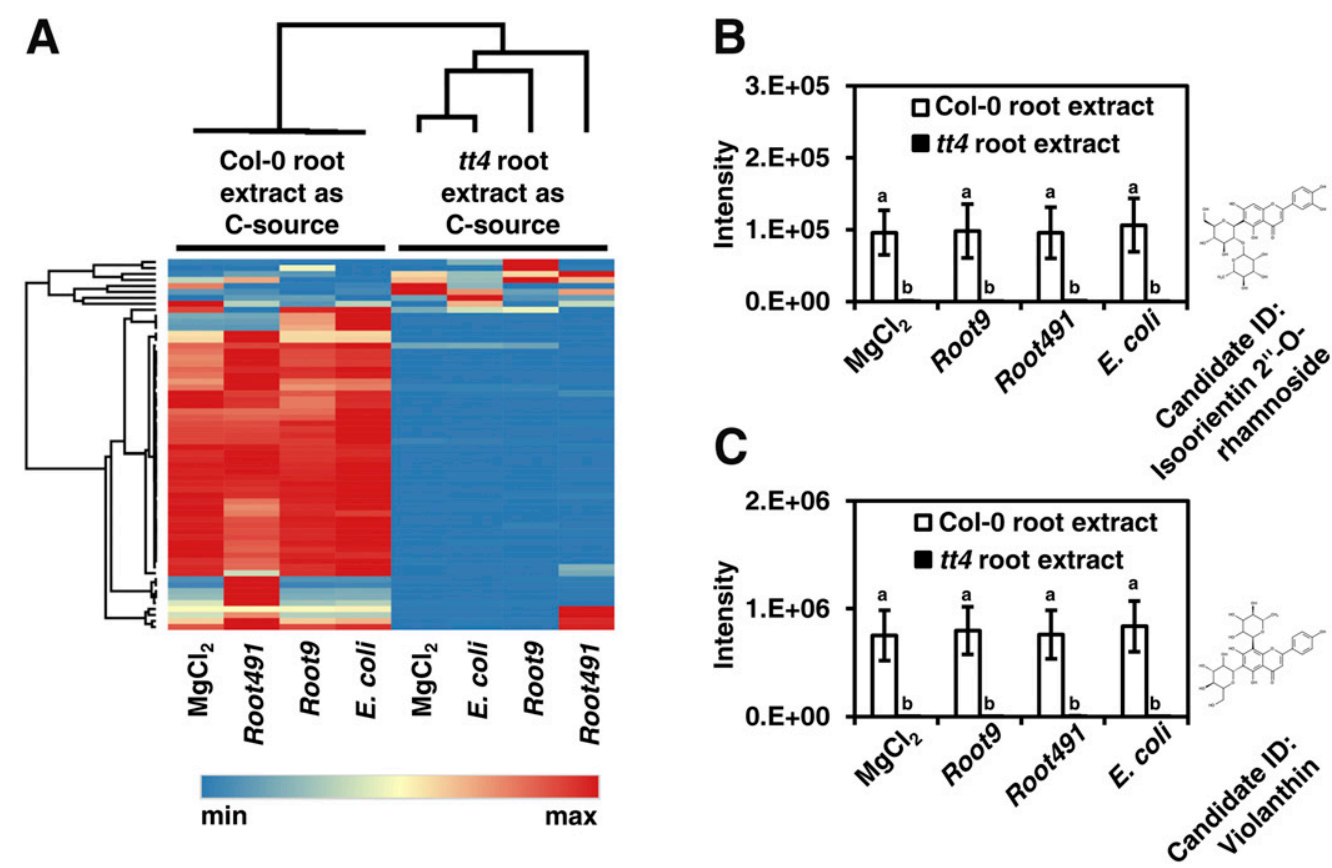

Fig. 8. Comparison of exometabolomic profiles between Col-0 versus $t t 4$. A, A heatmap depicting the mean intensity values, for 62 mass spectral (MS) features, after data were filtered to select for MS features that were differentially abundant between Col- 0 versus $t t 4$ in either the $\mathrm{MgCl}_{2}$ incubation or in any bacterial inoculation. Each row corresponds to a MS feature, and each column corresponds to a strain $\times$ medium combination. Rows and columns were hierarchically clustered using Pearson's correlation coefficient. B, and $\mathbf{C}$, Selected MS features likely to be flavonoid glycosides that differed significantly between Col-0 versus $t t 4$. Candidate metabolite identities (IDs) matched to isoorientin 2'-O-rhamnoside (B) and violanthin (C). White-filled columns represent mean feature intensity when Col-0 root extract was the sole carbon source, black-filled columns represent mean feature intensity when $t t 4$ root extract was the sole carbon source. $X$ axis labels indicate bacterial inoculation, with $\mathrm{MgCl}_{2}$ as a sterile control. Error bars represent standard deviation $(n=3)$. Different letters indicate significant difference at $P<0.01$ (Tukey's highly significant difference test). 
document a large number of MS features that the three bacterial strains release into two media, finding discrete patterns according to whether glucose or Col-0 root extract were provided as the sole carbon source. We found that $E$. coli gave out a large set of metabolites only under glucose nutrition, perhaps indicating that a metabolic bottleneck occurs under this single carbon substrate. Conversely, Rhizobium sp. Root 491 released a large set of metabolites only under Col-0 nutrition, some of which had $\mathrm{m} / \mathrm{z}$ values matching degradation products derived from consumption of specific root-derived metabolites. We also observe that Pseudomonas sp. Root9 releases a large array of nucleotides, which are likely to have roles in signaling. Our detection of these excreted metabolites shows that exometabolomic approaches applied to the rhizosphere have a strong potential to deepen our knowledge of microbial community function.

Confidently assigning identity to MS features detected via LC-MS is a longstanding problem in metabolomics research (Bowen and Northen 2010; Watson 2013). Here, we used the Metabosearch program to assign candidate identities to many of the MS features that were taken up or released by the studied strains, allowing us to postulate molecular mechanisms rather than simply comparing MS features. However, there are clear limitations to this annotation strategy, as it does not account for all possible fragments and adducts and, also, it does not differentiate between the many isomers that can be derived from a molecular formula. Obviously, it would be desirable to validate the identity of these MS features using reference compounds and tandem MS, but this approach is unfeasible for the large number of MS features detected in this analysis (1,982 features significant at $P<0.01$ between any two groups), particularly because our metabolite pool derives from the combined complexity of the plant plus bacterial metabolomes. Therefore, we refer to all our molecular assignations as candidate IDs and are designing future studies to focus on a smaller number of specific MS features with more analytical precision.

In the metabolomics literature, a wide variety of LC-MS methods have been applied to profile chemically diverse metabolites, with each method having various strengths and weaknesses (Gika et al. 2014). For instance, hydrophilic interaction liquid chromatography (HILIC) is better suited to resolve polar metabolites, whereas reverse-phase LC (RPLC) is better suited to resolve hydrophobic metabolites (Tang et al. 2016). Positive-mode ionization is better suited to detect a wide range of polar and nonpolar metabolites, whereas negative-mode ionization is better suited to detect specific metabolite classes, such as tricarboxylic acid cycle intermediates and phosphorylated metabolites (Theodoridis et al. 2012). Here, our methodology involved RPLC chromatography followed by positivemode MS and, although this gave good coverage of many diverse metabolites, there were still several metabolite classes that are not optimally resolved and detected by this method. For instance, sugars were poorly resolved with our chromatography setup and applying an alternative chromatography technique such as HILIC would yield higher-quality insights into sugar metabolism in the rhizosphere. Also, we did not detect molecules that only ionize in negative mode, such as glucosinolates, showing that alternative ionization conditions would be required to study microbial glucosinolate metabolism. Furthermore, organic acids are highly abundant in plant roots and exudates, and the microbial consumption of plant-derived organic acids is considered to be a major nutrient flux in the rhizosphere (Oburger et al. 2009; van Hees et al. 2005). However, our method seems poorly suited to detect organic acids, with only malate being observed in this study. Due to these limitations therefore, we encourage further studies that build on the general approach presented here, particularly those using other analytical methodologies to detect a wider range of molecules.
There is a large volume of literature studying how root exudation is involved in plant-microbe nutrient transfer (Hartmann et al. 2009; Massalha et al. 2017), and one potential criticism of our approach is that we have used root extracts rather than root exudates as the carbon source for bacterial growth. Here, we give four reasons to justify this choice. First, many rhizosphere microbes are root endophytes (Gaiero et al. 2013), and root metabolite extracts are probably a reasonable approximation of the endophytic substrate pool. Indeed, the root-inhabiting bacterial strains investigated in this study may, in fact, be endophytes, because the sampling procedures used by Bai et al. (2015) to generate these bacterial isolates did not discriminate between endophytes and epiphytes. Second, although root exudation is often used as a convenient shorthand to describe the belowground release of plant metabolites, rhizodeposition can occur through a range of mechanisms, such as i) lysis of sloughed-off root cells, ii) production of mucilage, iii) diffusion of volatiles, and iv) exudation of metabolites from living root cells (Dennis et al. 2010; Jones et al. 2009). Although estimates vary, results from several studies indicate that the amount of carbon deposited via root lysates can significantly outweigh what is deposited via root exudates (Farrar et al. 2003; Kaštovská et al. 2017). Indeed, the root metabolite extracts used in this study are probably a reasonable approximation of the metabolite pool that would occur upon initial lysis of sloughed-off root cells. Third, it is technically challenging to collect Arabidopsis root exudates in a representative, sterile, and reproducible manner (Jacoby et al. 2017; Vranova et al. 2013), particularly in the large quantities needed for this study. Fourth, there is considerable overlap between the molecules we observed in this study compared with the list of molecules reported to be present in root exudates, such as sugars, amino acids, organic acids, vitamins, nucleosides, and phenolics (Dakora and Phillips 2002; Mönchgesang et al. 2016). This seems reasonable, as any molecule that will eventually be exuded into the soil must first be present within the root cell. But despite these justifications for our approach, we accept that root exudates are still an important nutrient flux in the rhizosphere, particularly around rapidly proliferating meristematic tissue, and we encourage future studies that attempt to modify our approach to use root exudates as a sole carbon source.

It is well-documented that many microbial strains mediate plant growth promotion via mechanisms such as nutrient mobilization, pathogen suppression, and hormonal signaling (Bulgarelli et al. 2013), and there is increasing research interest in engineering the rhizospheric microbiome to promote these functions and thus replace synthetic agricultural inputs (Bender et al. 2016). However, any efforts to rationally design rhizospheric microbiomes are hampered by the fact that we have very little mechanistic understanding of the principles that govern microbial community formation in the rhizosphere (Busby et al. 2017). Here, we present an approach that could provide new mechanistic insights into rhizospheric microbial communities, by identifying the root-derived metabolites that are consumed by individual strains as carbon substrates and, also, the metabolites that are excreted by individual strains and, therefore, have possible roles in cross-feeding, signaling, or antibiosis. These insights have the potential to guide the rational design of high-functioning rhizosphere communities. For instance, plant varieties could be selected to contain root metabolite profiles that are tailored to nourish targeted microbial strains with desired functional benefits. To rationally guide microbial community formation, one possibility involves combining complementary strains with divergent substrate uptake profiles, which can coexist in the rhizosphere via niche differentiation. Another possibility involves the promotion of cross-feeding, by matching the metabolite release of one strain to the metabolite uptake of another strain. 


\section{MATERIALS AND METHODS}

Plant accessions and bacterial strains.

Plant genotypes used in this study were Arabidopsis thaliana ecotype Columbia (Col-0) and a $t t 4$ mutant derived from a T-DNA insertion in the chalcone synthase gene At5g13930, stock number SALK_020583. Both were purchased from the Nottingham Arabidopsis Stock Centre. Bacterial strains used in this study were Pseudomonas sp. Root9 (National Center for Biotechnology Information [NCBI] taxonomy ID 1736604), Rhizobium sp. Root491 (NCBI taxonomy ID 1736548), both isolated from field-grown Arabidopsis roots (Bai et al. 2015) and provided by P. Schulze-Lefert, Max Plank Institute for Plant Breeding Research, Cologne, Germany. Also used was Escherichia coli strain K12 MG1655 (NCBI taxonomy ID 511145), purchased from DSMZ.

\section{Plant cultivation, root metabolite extraction,} and total organic carbon (TOC) measurement.

Arabidopsis plants were cultivated using anchored shaking liquid culture, according to the method of Hétu et al. (2005). First, seeds were surface-sterilized for $2 \mathrm{~min}$ in $70 \%$ ethanol, followed by $5 \mathrm{~min}$ in $50 \%$ bleach, followed by rinsing five times in sterile Milli-Q water. Then, 15 to 20 seeds were pipetted onto steel mesh discs lying on agar plates $(0.7 \%$ agar, $1 \%$ sucrose, $0.5 \times$ Murashige and Skoog, pH 5.8) and were incubated at $4^{\circ} \mathrm{C}$ in darkness for 2 days. Next, plates were transferred to a growth cabinet, operating at $22^{\circ} \mathrm{C}$, with a $16-\mathrm{h}$ photoperiod, $120 \mu \mathrm{E}$ per square meter of light intensity for 7 days. Then, steel disks containing the anchored seedling plants were transferred to $250-\mathrm{ml}$ Erlenmeyer flasks, containing $15 \mathrm{ml}$ of growth medium (3\% sucrose, $0.5 \times$ Murashige and Skoog, pH 5.8), and were placed in a shaking incubator at $100 \mathrm{rpm}$ in the growth chamber. After 10 days on the sucrosecontaining medium, the medium was swapped to a medium without sucrose (0.5× Murashige and Skoog, pH 5.8), and plants were left shaking for 7 days. During shaking liquid cultivation, the medium was exchanged every three or four days. Plants were harvested by rinsing the plants twice in $10 \mathrm{mM} \mathrm{MgCl} 2$ and separating roots from shoots and immediately snap-freezing the root tissue in liquid $\mathrm{N}_{2}$. Root tissue was ground in liquid $\mathrm{N}_{2}$, using a mortar and pestle, and metabolites were extracted by adding $1 \mathrm{ml}$ of $90 \% \mathrm{MeOH}$ to $200 \mathrm{mg}$ of ground tissue, followed by a 30 -min incubation at $60^{\circ} \mathrm{C}$ with 1,500-rpm shaking. Extracts were then centrifuged at $20,000 \times g$ for $10 \mathrm{~min}$ and $850 \mu \mathrm{l}$ of supernatant was dried, using a vacuum centrifuge. Next, metabolite extracts were dissolved in Milli-Q water and were filter sterilized $(0.22 \mu \mathrm{m}$ pore size) (Millipore). TOC was measured using a Dimatoc 2000 (Dimatec).

\section{Formulation of bacterial growth media.}

Bacterial growth media was based on M9 formulation (Cold Spring Harbor Laboratory 2010). Nutrient concentrations were:

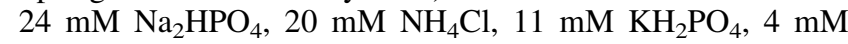
$\mathrm{NaCl}, 350 \mu \mathrm{M} \mathrm{MgSO}, 100 \mu \mathrm{M} \mathrm{CaCl} 2,50 \mu \mathrm{M}$ Fe-EDTA, $50 \mu \mathrm{M} \mathrm{H}_{3} \mathrm{BO}_{3}, 10 \mu \mathrm{M} \mathrm{MnCl}_{2}, 1.75 \mu \mathrm{M} \mathrm{ZnCl}_{2}, 1 \mathrm{uM} \mathrm{KI}, 800 \mathrm{nM}$ $\mathrm{Na}_{2} \mathrm{MoO}_{4}, 500 \mathrm{nM} \mathrm{CuCl}$, and $100 \mathrm{nM} \mathrm{CoCl}$. To this, one carbon source was added, either glucose $(10 \mathrm{mM}$, equivalent to $0.72 \mathrm{mg}$ of C per milliliter) or Arabidopsis root extract ( $0.72 \mathrm{mg}$ of $\mathrm{C}$ per milliliter, as measured by TOC).

\section{Bacterial cultivation and spent media collection.}

Bacterial strains were precultivated by streaking glycerol stocks onto trypticase soy agar plates $(0.5 \times$ trypticase soy broth [TSB], $1.2 \%$ agar) and incubation at $28^{\circ} \mathrm{C}$ for between 1 and 4 days. After visible growth, single colonies were picked from plates and inoculated into TSB medium $(0.5 \times$ TSB $)$ and were incubated for $24 \mathrm{~h}$ at $28^{\circ} \mathrm{C}$ with 200 -rpm shaking. Next, cells were harvested by centrifuging $800 \mu$ of culture at 5,000 $\times g$ for $2 \mathrm{~min}$ at room temperature. These cells were then rinsed three times in sterile $10 \mathrm{mM} \mathrm{MgCl}_{2}$ and resuspended at a final $\mathrm{OD}_{600}$ of 1.0 in sterile $10 \mathrm{mM} \mathrm{MgCl}_{2}$. For growth assays, $20 \mu \mathrm{l}$ of this bacterial suspension was inoculated into $380 \mu \mathrm{l}$ of growth medium, in individual wells of a sterile 48-well plate (Corning). These plates were then transferred to a plate reader (Tecan Infinite Pro 100) and were incubated at $28^{\circ} \mathrm{C}$ for $24 \mathrm{~h}$, with shaking ( 3 min continuous orbital shaking followed by $7 \mathrm{~min}$ stationary, shaking amplitude $3 \mathrm{~mm}$ ). Culture density at $\mathrm{OD}_{600}$ was measured once per 10-min cycle, without correcting for path length, and derived growth curves were analyzed using the Growthcurver program (Sprouffske and Wagner 2016). For spent media collection, bacterial cultures were centrifuged at $10,000 \times g$ for $10 \mathrm{~min}$ at room temperature; then, $250 \mu \mathrm{l}$ of supernatant was filtered, using a spin filter (Corning, $0.22 \mu \mathrm{m}$ pore size), and the flow-through was stored at $-80^{\circ} \mathrm{C}$. Three independent biological replicates were conducted, in which each replicate derived from an independent plant cultivation and also an independent bacterial cultivation. As a negative control (without bacteria), we included samples for which growth media were incubated with $20 \mu \mathrm{l}$ of sterile $10 \mathrm{mM} \mathrm{MgCl}_{2}$ instead of being inoculated with bacteria.

\section{LC-MS analysis of spent media.}

For spent media analyses, $2 \mu \mathrm{l}$ of sample was loaded onto a C18 column (XSelect HSS T3, $2.5 \mu$ m particle size, 10-nm pore size, $150 \mathrm{~mm}$ length by $3.0 \mathrm{~mm}$ width; Waters), using a highpressure LC (HPLC) (Dionex Ultimate 3000, Thermo Scientific). Buffer A was $0.1 \%$ FA in HPLC-grade water, buffer B was $0.1 \% \mathrm{FA}$ in HPLC-grade methanol, and flow rate was $500 \mu \mathrm{l} / \mathrm{min}$. Samples were eluted using the following gradient: hold at $1 \% \mathrm{~B}$ between 0 to $1 \mathrm{~min}$, linear increase to $40 \% \mathrm{~B}$ until $11 \mathrm{~min}$, linear increase to $99 \%$ B until $15 \mathrm{~min}$, hold at $99 \% \mathrm{~B}$ until $16 \mathrm{~min}$, linear decrease to $1 \% \mathrm{~B}$ until $17 \mathrm{~min}$, and finally, hold at $1 \%$ B until $20 \mathrm{~min}$. MS was conducted using a quadrupole-time-of-flight MS (maXis 4G; Bruker Daltonics), following ionization via an electrospray ionization source. The MS was operated in positive-ion mode, using $\mathrm{N}_{2}$ as drying gas at a flow rate of 8 liters $/ \mathrm{min}$, dry heater set to $220^{\circ} \mathrm{C}$, nebulizer pressure of $1.8 \mathrm{bar}$, capillary voltage of $4,500 \mathrm{~V}$, and collision radio frequency voltage of $370 \mathrm{~V}$ (corresponding to a mass range of 50 to $1,300 \mathrm{~m} / \mathrm{z}$ ). Scan rate was $1 \mathrm{~Hz}$.

\section{Data analysis.}

Raw .D files were centroided and converted to .MZMXL using the MSConvert program (Kessner et al. 2008). Files were then uploaded to XCMS online (Tautenhahn et al. 2012) and were aligned using the following parameters: $\mathrm{m} / \mathrm{z}$ tolerance of $15 \mathrm{ppm}$, minimum peak width of $10 \mathrm{~s}$, maximum peak width of $60 \mathrm{~s}$, signal/noise threshold of 10, overlapping peaks split when $\mathrm{m} / \mathrm{z}$ difference was greater than $0.01 \mathrm{~m} / \mathrm{z}$, features only considered if they occurred in at least five consecutive scans with an intensity greater than 5,000. Also, features were only considered if they occurred in at least two of three replicates from any sample group. The CAMERA algorithm was implemented to detect isotopes and adducts. Following export of files from XCMS online, data were filtered to remove isotopic peaks and to only include MS features with RT between 1 to $16 \mathrm{~min}$. Samples were compared between groups using Student's $t$ test, and the resulting $P$ values were corrected for multiple comparisons using the method of Storey (2002) to yield $q$ values. To calculate significant differences shown in Figures 4, 5, 6, 7, and 8 , data were compared using analysis of variance, followed by posthoc Tukey's highly significant difference test to determine 
significant differences between groups at $P<0.01$. To select the MS features that were taken up from Col- 0 media, we filtered for features with intensity $>10,000$ in Col-0 incubated with $\mathrm{MgCl}_{2}$, where $\log _{2}$ fold change was $>2$ between Col-0 incubated with $\mathrm{MgCl}_{2}$ versus Col-0 inoculated with bacteria and where the $t$ test of this comparison gave $P<0.01$. In instances in which the MS feature had an abundance of zero in the inoculated sample and, thus, no fold change was calculated, we imposed a filter that the feature must have a relative standard deviation of $<25 \%$ in the Col- $0 \mathrm{MgCl}_{2}$ sample. To select the MS features that were released into the media, we again filtered for features where $\log _{2}$ fold change was $>2$ between medium inoculated with bacteria versus medium incubated with $\mathrm{MgCl}_{2}$ and where the $t$ test of this comparison gave $P<0.01$. In instances in which the MS feature had an abundance of zero in the uninoculated sample and, thus, no fold change was calculated, we imposed a filter that the feature must have a relative standard deviation of $<25 \%$ in the sample inoculated with bacteria. To select the MS features that were differentially abundant between Col-0 and the $t t 4$ mutant, we filtered for MS features with a $\log _{2}$ fold change $>2$ and a $P$ value $<0.01$, either in the $\mathrm{MgCl}_{2}$ incubations or in any of the bacterial inoculations. To assign candidate metabolite identities for the MS features of interest, we used the Metabosearch program to search the $\mathrm{m} / \mathrm{z}$ values against three databases-MMCD, HMDB, and Lipidmaps (Zhou et al. 2012). We searched for possible $\mathrm{H}^{+}, \mathrm{Na}^{+}, \mathrm{K}^{+}$, and $\mathrm{NH}_{4}^{+}$adducts of each MS feature, imposing a $\pm 2 \mathrm{ppm} \mathrm{m} / \mathrm{z}$ error tolerance. In many cases, Metabosearch output multiple candidate identities per single MS feature, so to filter these assignments into a manageable list, we sorted for the metabolite hit with the lowest $\mathrm{m} / \mathrm{z}$ error and then for the metabolite hit with the lowest entry number in the KEGG database, and we present only the first result from this list.

\section{Data availability.}

All LC-MS data files have been uploaded to the Metabolights database (Haug et al. 2013).

\section{ACKNOWLEDGMENTS}

We thank P. Schulze-Lefert (Max Planck Institute for Plant Breeding, Cologne) for providing the root-inhabiting bacterial strains, V. Wewer and S. Metzger (CEPLAS MS Platform, University of Cologne) for performing LC-MS measurements, and N. Mantke (Institute of Geology and Mineralogy, University of Cologne) for performing TOC measurements.

\section{LITERATURE CITED}

Bai, Y., Muller, D. B., Srinivas, G., Garrido-Oter, R., Potthoff, E., Rott, M., Dombrowski, N., Münch, P. C., Spaepen, S., Remus-Emsermann, M., Hüttel, B., McHardy, A. C., Vorholt, J. A., and Schulze-Lefert, P. 2015. Functional overlap of the Arabidopsis leaf and root microbiota. Nature 528:364-369.

Baran, R., Brodie, E. L., Mayberry-Lewis, J., Hummel, E., Da Rocha, U. N., Chakraborty, R., Bowen, B. P., Karaoz, U., Cadillo-Quiroz, H., GarciaPichel, F., and Northen, T. R. 2015. Exometabolite niche partitioning among sympatric soil bacteria. Nat. Commun. 6:8289.

Barret, M., Morrissey, J. P., and O'Gara, F. 2011. Functional genomics analysis of plant growth-promoting rhizobacterial traits involved in rhizosphere competence. Biol. Fertil. Soils 47:729-743.

Beckers, B., Op De Beeck, M., Weyens, N., Boerjan, W., and Vangronsveld, J. 2017. Structural variability and niche differentiation in the rhizosphere and endosphere bacterial microbiome of field-grown poplar trees. Microbiome 5:25.

Bender, S. F., Wagg, C., and van der Heijden, M. G. A. 2016. An underground revolution: Biodiversity and soil ecological engineering for agricultural sustainability. Trends Ecol. Evol. Amst. 31:440-452.

Benizri, E., Dedourge, O., Dibattista-Leboef, C., Piutti, S., Nguyen, C., and Guckert, A. 2002. Effect of maize rhizodeposits on soil microbial community structure. Appl. Soil Ecol. 21:261-265.
Bochner, B. R., Gadzinski, P., and Panomitros, E. 2001. Phenotype microarrays for high-throughput phenotypic testing and assay of gene function. Genome Res. 11:1246-1255.

Bowen, B. P., and Northen, T. R. 2010. Dealing with the unknown: Metabolomics and metabolite atlases. J. Am. Soc. Mass Spectrom. 21: 1471-1476.

Bulgarelli, D., Garrido-Oter, R., Münch, P. C., Weiman, A., Dröge, J., Pan, Y., McHardy, A. C., and Schulze-Lefert, P. 2015. Structure and function of the bacterial root microbiota in wild and domesticated barley. Cell Host Microbe 17:392-403.

Bulgarelli, D., Schlaeppi, K., Spaepen, S., van Themaat, E. V. L., and Schulze-Lefert, P. 2013. Structure and functions of the bacterial microbiota of plants. Ann. Rev. Plant Biol. 64:807-838.

Busby, P. E., Soman, C., Wagner, M. R., Friesen, M. L., Kremer, J., Bennett, A., Morsy, M., Eisen, J. A., Leach, J. E., and Dangl, J. L. 2017. Research priorities for harnessing plant microbiomes in sustainable agriculture. PLoS Biol. 15:e2001793.

Chi, F., Shen, S. H., Cheng, H. P., Jing, Y. X., Yanni, Y. G., and Dazzo, F. B. 2005. Ascending migration of endophytic rhizobia, from roots to leaves, inside rice plants and assessment of benefits to rice growth physiology. Appl. Environ. Microbiol. 71:7271-7278.

Compant, S., Clement, C., and Sessitsch, A. 2010. Plant growth-promoting bacteria in the rhizo- and endosphere of plants: Their role, colonization, mechanisms involved and prospects for utilization. Soil Biol. Biochem. 42:669-678.

Cold Spring Harbor Laboratory. 2010. Recipe: M9 minimal medium (standard). Cold Spring Harbor Prot. 2010(8):pdb.rec12295.

Dakora, F. D., and Phillips, D. A. 2002. Root exudates as mediators of mineral acquisition in low-nutrient environments. Plant Soil 245:35-47.

Dennis, P. G., Miller, A. J., and Hirsch, P. R. 2010. Are root exudates more important than other sources of rhizodeposits in structuring rhizosphere bacterial communities? FEMS Microbiol. Ecol. 72:313-327.

Edwards, J., Johnson, C., Santos-Medellín, C., Lurie, E., Podishetty, N. K., Bhatnagar, S., Eisen, J. A., and Sundaresan, V. 2015. Structure, variation, and assembly of the root-associated microbiomes of rice. Proc. Natl. Acad. Sci. U.S.A. 112:E911-E920.

Egli, T. 2015. Microbial growth and physiology: A call for better craftsmanship. Front. Microbiol. 6:287.

Enjalbert, B., Letisse, F., and Portais, J.-C. 2013. Physiological and molecular timing of the glucose to acetate transition in Escherichia coli. Metabolites 3:820-837.

Farrar, J., Hawes, M., Jones, D., and Lindow, S. 2003. How roots control the flux of carbon to the rhizosphere. Ecology 84:827-837.

Fei, F., Dicenzo, G. C., Bowdish, D. M. E., McCarry, B. E., and Finan, T. M. 2016. Effects of synthetic large-scale genome reduction on metabolism and metabolic preferences in a nutritionally complex environment. Metabolomics 12:23.

Foster, K. R., Schluter, J., Coyte, K. Z., and Rakoff-Nahoum, S. 2017. The evolution of the host microbiome as an ecosystem on a leash. Nature 548:43-51.

Gaiero, J. R., McCall, C. A., Thompson, K. A., Day, N. J., Best, A. S., and Dunfield, K. E. 2013. Inside the root microbiome: Bacterial root endophytes and plant growth promotion. Am. J. Bot. 100:1738-1750.

García-Fraile, P., Carro, L., Robledo, M., Ramírez-Bahena, M. H., FloresFélix, J. D., Fernández, M. T., Mateos, P. F., Rivas, R., Igual, J. M., Martínez-Molina, E., Peix, Á., and Velázquez, E. 2012. Rhizobium promotes non-legumes growth and quality in several production steps: Towards a biofertilization of edible raw vegetables healthy for humans. PLoS One 7:e38122.

Gika, H. G., Theodoridis, G. A., Plumb, R. S., and Wilson, I. D. 2014. Current practice of liquid chromatography-mass spectrometry in metabolomics and metabonomics. J. Pharm. Biomed. Anal. 87:12-25.

Goldfarb, K. C., Karaoz, U., Hanson, C. A., Santee, C. A., Bradford, M. A., Treseder, K. K., Wallenstein, M. D., and Brodie, E. L. 2011. Differential growth responses of soil bacterial taxa to carbon substrates of varying chemical recalcitrance. Front. Microbiol. 2:94.

Grundling, A., and Lee, V.T. 2016. Old concepts, new molecules and current approaches applied to the bacterial nucleotide signalling field. Philos. T. Roy. Soc. B 371. Published online. doi: https://doi.org/10. 1098/rstb.2015.0503.

Harder, W., and Dijkhuizen, L. 1982. Strategies of mixed substrate utilization in microorganisms. Philos. Trans. R. Soc. Lond. B Biol. Sci. 297:459-480.

Hartmann, A., Schmid, M., van Tuinen, D., and Berg, G. 2009. Plant-driven selection of microbes. Plant Soil 321:235-257.

Hassan, S., and Mathesius, U. 2012. The role of flavonoids in root-rhizosphere signalling: Opportunities and challenges for improving plant-microbe interactions. J. Exp. Bot. 63:3429-3444. 
Haug, K., Salek, R. M., Conesa, P., Hastings, J., de Matos, P., Rijnbeek, M., Mahendraker, T., Williams, M., Neumann, S., Rocca-Serra, P., Maguire, E., González-Beltrán, A., Sansone, S. A., Griffin, J. L., and Steinbeck, C. 2013. MetaboLights-An open-access general-purpose repository for metabolomics studies and associated meta-data. Nucleic Acids Res. 41 (D1):D781-D786

Hétu, M.F., Tremblay, L.J., and Lefebvre, D.D. 2005. High root biomass production in anchored Arabidopsis plants grown in axenic sucrose supplemented liquid culture. Biotechniques39:345-349.

Jacoby, R., Peukert, M., Succurro, A., Koprivova, A., and Kopriva, S. 2017. The role of soil microorganisms in plant mineral nutrition-current knowledge and future directions. Front. Plant Sci. 8:1617.

Jones, D. L., Nguyen, C., and Finlay, R. D. 2009. Carbon flow in the rhizosphere: Carbon trading at the soil-root interface. Plant Soil 321:5-33.

Kaštovská, E., Edwards, K., and Santruckova, H. 2017. Rhizodeposition flux of competitive versus conservative graminoid: Contribution of exudates and root lysates as affected by N loading. Plant Soil 412:331-344.

Kessner, D., Chambers, M., Burke, R., Agus, D., and Mallick, P. 2008. ProteoWizard: Open source software for rapid proteomics tools development. Bioinformatics 24:2534-2536

Knee, E. M., Gong, F. C., Gao, M., Teplitski, M., Jones, A. R., Foxworthy, A., Mort, A. J., and Bauer, W. D. 2001. Root mucilage from pea and its utilization by rhizosphere bacteria as a sole carbon source. Mol. PlantMicrobe Interact 14:775-784.

Lundberg, D.S., Lebeis, S.L., Paredes, S.H., Yourstone, S., Gehring, J., Malfatti, S., Tremblay, J., Engelbrektson, A., Kunin, V., del Rio, T. G., Edgar, R. C., Eickhorst, T., Ley, R. E., Hugenholtz, P., Tringe, S. G., and Dangl, J. L. 2012. Defining the core Arabidopsis thaliana root microbiome. Nature 488:86-90.

Massalha, H., Korenblum, E., Tholl, D., and Aharoni, A. 2017. Small molecules below-ground: The role of specialized metabolites in the rhizosphere. Plant J. 90:788-807.

McCully, M. E. 2001. Niches for bacterial endophytes in crop plants: A plant biologist's view. Aust. J. Plant Physiol. 28:983-990.

Mönchgesang, S., Strehmel, N., Schmidt, S., Westphal, L., Taruttis, F., Müller, E., Herklotz, S., Neumann, S., and Scheel, D. 2016. Natural variation of root exudates in Arabidopsis thaliana-linking metabolomic and genomic data. Sci. Rep. 6:29033.

Monod, J. 1966. From enzymatic adaptation to allosteric transitions. Science 154:475-483.

Müller, D. B., Vogel, C., Bai, Y., and Vorholt, J. A. 2016. The plant microbiota: Systems-level insights and perspectives. Ann. Rev. Genet. 50:211-234. Annual Reviews, Palo Alto.

Oburger, E., Kirk, G. J. D., Wenzel, W. W., Puschenreiter, M., and Jones, D. L. 2009. Interactive effects of organic acids in the rhizosphere. Soil Biol. Biochem. 41:449-457.

Peiffer, J. A., Spor, A., Koren, O., Jin, Z., Tringe, S. G., Dangl, J. L., Buckler, E. S., and Ley, R. E. 2013. Diversity and heritability of the maize rhizosphere microbiome under field conditions. Proc. Natl. Acad. Sci. U.S.A. 110:6548-6553.

Pinu, F. R., and Villas-Boas, S. G. 2017. Extracellular microbial metabolomics: The state of the art. Metabolites 7:43.

Ryffel, F., Helfrich, E. J. N., Kiefer, P., Peyriga, L., Portais, J. C., Piel, J., and Vorholt, J. A. 2016. Metabolic footprint of epiphytic bacteria on Arabidopsis thaliana leaves. ISME J. 10:632-643.
Sasse, J., Martinoia, E., and Northen, T. 2018. Feed your friends: Do plant exudates shape the root microbiome? Trends Plant Sci. 23:25-41.

Silva, L. P., and Northen, T. R. 2015. Exometabolomics and MSI: Deconstructing how cells interact to transform their small molecule environment. Curr. Opin. Biotechnol. 34:209-216.

Sprouffske, K., and Wagner, A. 2016. Growthcurver: An R package for obtaining interpretable metrics from microbial growth curves. BMC Bioinformatics 17:172.

Storey, J. D. 2002. A direct approach to false discovery rates. J. R. Stat. Soc. B 64:479-498.

Szoboszlay, M., White-Monsant, A., and Moe, L. A. 2016. The effect of root exudate $7,4^{\prime}$-dihydroxyflavone and naringenin on soil bacterial community structure. PLoS One 11:e0146555.

Tang, D. Q., Zou, L., Yin, X. X., and Ong, C. N. 2016. HILIC-MS for metabolomics: An attractive and complementary approach to RPLC-MS. Mass Spectrom. Rev. 35:574-600.

Tautenhahn, R., Patti, G. J., Rinehart, D., and Siuzdak, G. 2012. XCMS Online: A web-based platform to process untargeted metabolomic data. Anal. Chem. 84:5035-5039.

Theodoridis, G. A., Gika, H. G., Want, E. J., and Wilson, I. D. 2012. Liquid chromatography-mass spectrometry based global metabolite profiling: A review. Anal. Chim. Acta 711:7-16.

van Hees, P. A. W., Jones, D. L., Finlay, R., Godbold, D. L., and Lundstomd, U. S. 2005. The carbon we do not see-The impact of low molecular weight compounds on carbon dynamics and respiration in forest soils: A review. Soil Biol. Biochem. 37:1-13.

Vogels, G. D., and Van der Drift, C. 1976. Degradation of purines and pyrimidines by microorganisms. Bacteriol. Rev. 40:403-468.

Vranova, V., Rejsek, K., Skene, K. R., Janous, D., and Formanek, P. 2013. Methods of collection of plant root exudates in relation to plant metabolism and purpose: A review. J. Plant Nutr. Soil Sci. 176:175-199.

Watson, D. G. 2013. A rough guide to metabolite identification using high resolution liquid chromatography mass spectrometry in metabolomic profiling in metazoans. Comput. Struct. Biotechnol. J. 4: e201301005.

Werner, A. K., and Witte, C. P. 2011. The biochemistry of nitrogen mobilization: Purine ring catabolism. Trends Plant Sci. 16:381-387.

Widder, S., Allen, R. J., Pfeiffer, T., Curtis, T. P., Wiuf, C., Sloan, W. T., Cordero, O. X., Brown, S. P., Momeni, B., Shou, W., Kettle, H., Flint, H. J., Haas, A. F., Laroche, B., Kreft, J. U., Rainey, P. B., Freilich, S., Schuster, S., Milferstedt, K., van der Meer, J. R., Großkopf, T., Huisman, J., Free, A., Picioreanu, C., Quince, C., Klapper, I., Labarthe, S., Smets, B. F., Wang, H., and Soyer, O. S. 2016. Challenges in microbial ecology: Building predictive understanding of community function and dynamics. ISME J. 10:2557-2568.

Womack, J. E., and O'Donovan, G. A. 1978. Orotic acid excretion in some wild-type strains of Escherichia coli K-12. J. Bacteriol. 136: 825-827.

Zhou, B., Wang, J., and Ressom, H. W. 2012. MetaboSearch: Tool for massbased metabolite identification using multiple databases. PLoS One 7: e40096.

\section{AUTHOR-RECOMMENDED INTERNET RESOURCE}

Metabolights study: http://www.ebi.ac.uk/metabolights/MTBLS555 